\title{
REVIEWS
}

Adv Clin Exp Med 2015, 24, 6, 1113-1117

DOI: $10.17219 /$ acem $/ 50572$

(c) Copyright by Wroclaw Medical University

ISSN 1899-5276

\begin{abstract}
MirosŁaw SŁowik ${ }^{1, A}$, D, Monika M. Biernat ${ }^{2,3, \text { B, D, Donata Urbaniak-Kujda }}$ 3, B, E, Katarzyna Kapelko-S ŁowiK ${ }^{3, A, ~ B, ~ D, ~ M a r t a ~ M i s i u k-H o j Ł o l, ~ F ~}$
\end{abstract}

\section{Mycotic Infections of the Eye}

${ }^{1}$ Department and Clinic of Ophthalmology, Wroclaw Medical University, Poland

${ }^{2}$ Department of Microbiology, Wroclaw Medical University, Poland

${ }^{3}$ Department and Clinic of Hematology, Blood Neoplasms and Bone Marrow Transplantation, Wroclaw Medical University, Poland

A - research concept and design; B - collection and/or assembly of data; C - data analysis and interpretation;

$\mathbf{D}$ - writing the article; $\mathbf{E}$ - critical revision of the article; $\mathbf{F}$ - final approval of article

\begin{abstract}
Fungal infections of the eye are an important cause of significant visual loss and blindness in some regions of the world, especially developing countries. Ocular mycoses remain a diagnostic and therapeutic challenge to the ophthalmologist. Corneal infection is the most frequent presentation, but the orbit, eyelids, lacrimal apparatus, conjunctiva, sclera and internal structures of the eye can also be affected. Candida spp., Fusarium spp. and Aspergillus spp. are the most frequently isolated organisms in fungal keratitis and in endophthalmitis. The difficulties posed by ocular mycoses are mainly related to establishing the clinical diagnosis, isolation of the fungal pathogen and effective local treatment, particularly in infections of the cornea. The critical issue in diagnosing fungal infection of the eye is microbiological identification of the etiologic agent in clinical samples. Early diagnosis and prompt treatment allow serious complications, including blindness, to be avoided. Local, systemic and even surgical treatment is applied in the therapy (Adv Clin Exp Med 2015, 24, 6, 1113-1117).
\end{abstract}

Key words: ocular mycoses, fungal keratitis, antifungal drugs.

Fungal infections of the eye are considered an important cause of significant loss of vision in some regions of the world, especially in Asia, where they are the main causes of blindness [1]. The inflammatory process typically involves the cornea, but the orbit, eyelids, lacrimal apparatus, conjunctiva, sclera and internal structures of the eye can also be affected. Ocular infections of fungal etiology still remain a diagnostic and therapeutic challenge for the ophthalmologist. The difficulties are mainly related to establishing the clinical diagnosis, isolation of the fungal pathogen and effective local treatment, particularly in infections of the cornea. The negative predictive factors include delayed diagnosis, resulting from a lack of suspicion of fungal etiology, and inadequate initial treatment. Poor penetration of antifungal drugs to the eye tissue and the poor commercial availability of drug formulations are also problematic.

\section{Eyelids}

Inflammation of the eyelids in most cases has a bacterial etiology but fungi can also infect the superficial and deeper layers of the eyelid. The inflammatory lesions may be ulcerative, as in the course of infections caused by Cryptococcus spp., or productive, as in the course of blastomycosis. A case of a man with necrotic fasciitis of the eyelids caused by Cryptococcus neoformans after a trivial trauma by a splinter of wood has been documented [2]. Blepharitis caused by Rhinosporidium seeberi can also be observed; the most frequent location of this endemic infection is the conjunctiva [3]. Candida spp. infections usually appear in patients treated with broad-spectrum antibiotics or immunosuppressive drugs [4]. Changes in the eyelids characterized as an increasing nodule with a central ulcer, sometimes coexisting with keratoconjunctivitis, have been reported in more than 
$50 \%$ of cases of ocular paracoccidioidomycosis; this infection is seen mainly in men over 30 years of age, employed in agriculture in endemic areas [5]. In this infection, typical changes are observed within the eyelids and require not only the use of antifungal drugs but also surgical removal of fibrotic lesions.

\section{Tear Ducts}

Fungal infection of the tear ducts is very rare. A very high risk factor for this infection is local therapy with antibiotics or corticosteroids [6]. Infection of the tear ducts is often accompanied by conjunctivitis. A lack of response to antibiotic or corticosteroid treatment raises the suspicion of fungal etiology. Natamycin drops have been used in local therapy, as well as rinsing the tear ducts and the lacrimal sac with a solution of amphotericin B or nystatin [7]. If there is no response to treatment, incision of the lacrimal ducts has been used. To reconstruct the lacrimal channels, intubation using silicone tubes can be used. Fungal etiology is responsible for about $5 \%$ of cases of lacrimal sac infection. The main pathogens include Aspergillus spp. and Candida spp., but cases caused by Rhizopus spp. have been reported as well [8]. Fungi usually cause chronic infections, where the main symptom is lachrymation accompanied by conjunctivitis and edema of the medial angle of the eyelid. Moreover, compression of this region causes the presence of a purulent discharge in the lacrimal point of the lower eyelid. Treatment often requires surgical removal of the lacrimal sac or anastomosis of the lacrimal sac with the mucosa of the nasal cavity [8].

\section{Conjunctiva}

The prevalence of fungal conjunctivitis is low. In India, Sri Lanka and the Balkans, endemic infections caused by Rhinosporidium have been reported $[3,9]$. Closed reservoirs of water constitute an important risk factor. Fungal conjunctivitis is usually secondary to inflammation of the cornea, lacrimal sac and tear ducts. The etiologic agents include fungi of the genera Candida spp., Aspergillus spp. Sporotrichum spp., Blastomyces spp., Coccidioides spp., Malassezia spp. and dermatophytes (Microsporum, Trichophyton, Epidermophyton), which cause the symptoms of acute inflammation of the conjunctiva with mucopurulent discharge. The infected lesions usually have a productive character with a grayish deposit on the conjunctiva with further formation of eyelid-eyeball adhesions (in the case of a Candida infection) or papular changes of a white-yellow color (Sporotrichum spp.). In the case of an infection caused by Rhinosporidium, pink or red inflammatory changes have been observed, which may be pedicled and are usually located primarily in the palpebral conjunctiva [10]. Fungal conjunctivitis causes serious complications and requires intensive antifungal therapy; in the case of a Rhinosporidium infection, surgical debridement is also necessary.

\section{Sclera}

Fungal sclera changes are rare but have important clinical significance. Their occurrence has been reported in the course of inflammation of the cornea (caused by Absidia corymbifera, Acremonium spp. or Lasiodiplodia theobromare) [11, 12], in traumas (caused by Aspergillus spp. or Sporothrix schenckii), [13], as a complication of eye surgery, and as an endogenous infection. Appropriate therapeutic management is not clear, and success has only been obtained in some cases. Surgical debridement may also be needed.

\section{Cornea}

Inflammation of the cornea of fungal etiology manifests as purulent, ulcerative infection. According to different authors, this form of the disease may be responsible for $6 \%$ to $53 \%$ of cases of ulcerative keratitis [1]. The most frequent risk factors include trauma, long-term use of topical corticosteroids and antibiotics, systemic diseases, especially diabetes, pre-existing eye surface infections and contact lenses $[1,14]$. Molds, such as Fusarium spp. and Aspergillus spp., are the predominant causes of fungal infections of the cornea in most parts of the world. They are found most frequently in young healthy men who work in the open air, such as farmers. Trauma factors include particles of mud or dust, tree branches, metal particles, plant-derived organic matter and the use of nylon-line brushcutters. Candida spp. is the most common etiologic agent of keratitis [14]. Fungal infections of the cornea can develop in people who previously suffered from ulcers of the stromal corneal epithelium as a result of infection with the Herpes simplex virus or the use of contact lenses. Fungal infections can occur in people with systemic diseases as well as in pre-existing diseases of the eye surface. Rosa et al. compared different kinds of fungi (filamentous fungi and yeasts) as risk factors in fungal keratitis; they stated that injuries of the eye favored infections caused by Fusarium 
spp. (70\%), Curvularia spp. (11\%), or Aspergillus spp. (5\%), whereas diabetes predisposed to infections caused by Fusarium spp. (67\%) and Candida spp. (13\%). Chronic use of topical ophthalmic drugs may lead to infections caused by Candida spp. (44\%) and Fusarium spp. (38\%); in contrast, in patients treated with topical corticosteroids, the most frequently isolated species are Candida spp., Aspergillus spp., Acremonium spp. and Curvularia spp. (22\% each) [15]. Topically applied 5\% natamycin is used as first line treatment in superficial corneal inflammation caused by filamentous fungi, whereas $0.15 \%$ amphotericin B or $1 \%$ econazole is used in Candida infections. Mechanical removal of the corneal epithelium over the pathological change facilitates the penetration of the drugs. The oral azoles - voriconazole, itraconazole and fluconazole - are used additionally if the inflammatory process involves deeper layers [16]. In severe cases, usually with the presence of pus in the anterior chamber of the eye, fluconazole can be used in subconjunctival injections. Tunnel boring keratoplasty is used in non-responders and in cases when corneal perforations are already present [16].

\section{Uveitis and Endophthalmitis}

Bloodstream infections are a common cause of inflammation of the uveal tract. The risk factors include catheters, AIDS, neutropenia and immunosuppressive therapy. Most cases are caused by Candida spp.; other pathogens are Cryptococcus spp., Sporothrix schenckii and Blastomyces spp. [16]. Multifocal uveitis has been observed on the fundus of the eye, and deterioration of vision can be caused by changes located centrally on the fundus of the eye or accompanied by more severe vitritis. In infections caused by Cryptococcus neoformans, inflammation of the iris and cornea may also be present. Treatment with intravenous amphotericin B or with oral azoles (fluconazole, voriconazole or itraconazole) is indicated. Vitrectomy with the intravitreal administration of amphotericin B and voriconazole is used in infections accompanied by inflammation of the vitreous body [16].

Presumed ocular histoplasmosis syndrome is a fungal infection caused by Histoplasma capsulatum, characterized by diffuse, multifocal choroidal lesions, some of which become scarred when they are no longer active. The course of the disease is usually asymptomatic, but a reduction in visual acuity may occasionally occur; those cases are associated with choroidal neovascularization of the subretinal membranes of lesions located in the macula. In therapy, surgical removal of choroidal membranes may be needed. In recent years, there have been reports of positive results from anti-vascular endothelial growth factor (VEGF) therapy in intravitreal injections [17].

Fungi can cause both endogenous and exogenous endophthalmitis. Endogenous infections may be due to complications of fungal inflammations located in other organs, such as the heart valves, the central nervous system or the genitourinary tract. Candidosis is present mainly in people with weakened immune system, treated for sepsis with broad-spectrum antibiotics or nourished parenterally, as well as in immunocompromised patients after organ transplantation. Aspergillus spp. and Fusarium spp. are the most common causes of endogenous infection in drug addicts and people undergoing long-term corticosteroid therapy $[18,19]$. The drug of choice is voriconazole [20]. Surgical removal of the vitreous (vitrectomy) is used in patients who do not respond to standard anti-fungal treatment [21].

Exogenous endophthalmitis occurs after trauma, intraocular surgery or as a complication of corneal inflammation. Infection caused by Candi$d a$ spp. is diagnosed predominantly after surgery, whereas fusariosis appears after injury and inflammation of the cornea. Infections caused by Paecilomyces, Aspergillus spp., Acremonium, Exophiala, Pseudallescheria, Scytalidium, Sporothrix spp. and Penicillium spp. have also been reported [21]. Diagnosis should be based primarily on cultures of the intraocular fluid, which in most cases are positive. In treating endophthalmitis due to Candida spp., without involvement of the vitreous body, the use of intravenous fluconazole or voriconazole is recommended. Beneficial effects of a combination of flucytosine with amphotericin B have also been reported. However, in candidosis and aspergillosis where the inflammatory process includes not only the choroid and retina but also the vitreous, the standard intravenous treatment should be further complemented by intravitreal voriconazole and amphotericin B. The vitrectomy procedure is used in those patients who do not respond to conservative treatment [22].

\section{Eye Socket}

Infections of the eye socket are mostly a consequence of orbital inflammation in the surrounding tissues, such as the paranasal sinuses, skin and nasopharynx. Fungal infections of the tissues of the eye socket are significantly less common than bacterial infections, and they usually occur in people with poorly controlled diabetes, in immunocompromised patients with AIDS, in cancer patients, or in subjects who are taking long-term 
corticosteroids or immunosuppressive therapy [9]. The etiologic factors may include Mucor, Rhizopus, Absidia, Aspergillus spp., Blastomyces and Sporothrix spp. Prompt diagnosis and effective treatment of the primary disease are extremely important for the future prognosis. The therapy should be based on wide excision of the necrotic tissue early in the course of the disease and the implementation of antifungal drugs [23]. Liposomal amphotericin B used intravenously is usually effective. In severe cases, surgical removal of pathologically changed orbital tissues and pansinusectomy may be necessary [24].

\section{Diagnostics}

The critical issue for diagnosing fungal infections of the eye is microbiological identification of the etiologic agent in clinical samples, including swabs from the conjunctival sac or tissue material (corneal scrapings, vitreous or other material obtained during biopsy). Smears of clinical samples stained by the Gram stain method to identify Candida spp., by methyl blue for the presence of filamentous fungi and India ink staining for Cryptococcus spp. can be used in routine diagnosis using light microscopy. Tissue materials such as corneal scrapings can be lightened with dimethyl sulfoxide (DMSO), which allows better visualization of the fungus. In the culture methods, the samples are inoculated on Sabouraud medium and incubated at $37^{\circ} \mathrm{C}$ and $27^{\circ} \mathrm{C}$ for $7-10$ days. The plates are examined daily followed by macroscopic and microscopic evaluation of the colonies. Other diagnostic methods include the detection of specific antigens, such as the mannan antigen of Candida, the galactomannan antigen of Aspergillus and the cryptococcal antigen in serum samples. Molecular techniques, such as fluorescence in situ hybridization (FISH), polimerase chain reaction (PCR) and reverse transcription-PCR (RT-PCR) for the detection of genetic material of the fungi in clinical specimens can also be used. Histopathological examinations of tissue samples are always of great value in the diagnosis of invasive fungal infections, but are not always possible. High-resolution computed tomography imaging (HRCT) and magnetic resonance imaging (MR), which examine characteristic images changing dynamically in the course of the disease, can also be very useful [25].

\section{Summary}

Fungal infections continue to be important causes of eye diseases. In Asia, fungal infections of the cornea are the major cause of blindness. Candida spp., Fusarium spp., and Aspergillus spp. are the most common pathogens responsible for corneal infections and mycoses of the eyeball. An increased risk of fungal infections is associated with immunodeficiency (e.g. AIDS), diabetes, surgery, antibiotic and corticosteroid therapy as well as drug addiction. Early diagnosis and rapid aggressive antifungal therapy have a significant impact on the course of the disease and may reduce complications, including blindness.

\section{References}

[1] Ayse K, Sengul O: Ocular Fungal Infections. Curr Eye Res 2011, 36, 179-189.

[2] Doorenbos-Bot AC, Hooymans JM, Blanksma LJ: Periorbital necrotising fasciitis due to Cryptococcus neoformans in a healthy young man. Doc Ophthalmol 1990, 75, 315-320.

[3] Ganne P, Babu R, Mohan Girija G: Conjunctival rhinosporidiosis. JAMA Ophthalmol 2015, Jan, 133, 39.

[4] Duncan K, Jeng BH: Medical management of blepharitis. Curr Opin Ophthalmol 2015, 26, 289-294.

[5] Cruz AA, Zenha F, Silva JT Jr, Martinez R: Eyelid involvement in paracoccidioidomycosis. Ophthal Plast Reconstr Surg 2004, 20, 212-216.

[6] Różycki R, Rekas M, Wiśniewski P: Mycotic lacrimal canaliculitis - cases report. Klin Oczna 2004, 106, 338-342.

[7] Behrens-Baumann W: Mycoses of the eye and its adnexa. Dev Ophthalmol 1999, 32, $27-107$.

[8] Ghose S, Mahajan VM: Fungal flora in congenital dacryocystitis. Indian J Ophthalmol 1990, 38, 189-190.

[9] Philips R, Adamski Z: Zakażenia grzybicze oka. W: Mikologia lekarska dla lekarzy i studentów. Wyd. Nauk. Uniwersytetu Medycznego, Poznań 2007, $2^{\text {nd }}$ ed., 236-248.

[10] Suh LH, Barron J, Dubovy SR, Gaunt ML, Ledee DR, Miller D, Fell JW, Forster RK: Ocular rhinosporidiosis presenting as chronic follicular conjunctivitis in a contact lens wearer. Arch Ophthalmol 2009, 127, $1076-1077$.

[11] Marshall DH, Brownstein S, JacksonWB: Post-traumatic corneal mucormycosis caused by Absidia corymbifera. Ophthalmology 1997, 104, 1107-1111.

[12] Borderie VM, Bourcier TM, Poirot JLP: Endophthalmitis after Lasiodiplodia theobromae corneal abscess. Graefe's Arch Clin Exp Ophthalmol 1997, 235, 259-261.

[13] Fincher T, Fulcher SF: Diagnostic and therapeutic challenge of Aspergillus flavus scleritis. Cornea 2007, 26, 618-620.

[14] Khater MM, Shehab NS, El-Badry AS: Comparison of mycotic keratitis with nonmycotic keratitis: an epidemiological study. J Ophthalmol 2014, 2014, 254302. DOI: 10.1155/2014/254302. Epub 2014 Dec 7.

[15] Rosa RH, Miller D, Alfonso EC: The changing spectrum of fungal keratitis in South Florida. Ophthalmology 1994, 101, 1005-1013. 
[16] Kański JJ, Bowling B: Okulistyka kliniczna. Elsevier Uraban \& Partner, Wrocław, 2013, $4^{\text {th }}$ ed.

[17] Nielsen JS, Fick TA, Saggau DD, Barnes CH: Intravitreal anti-vascular endothelial growth factor therapy for choroidal neovascularization secondary to ocular histoplasmosis syndrome. Retina 2012, 32, 468-472.

[18] Lamaris GA, Esmaeli B, Chamilos G: Fungal endophthalmitis in a tertiary care cancer center: A review of 23 cases. Eur J Clin Microbiol Infect Dis 2008, 27, 343-347.

[19] Mody KH, Ali MJ, Vemuganti GK, Nalamada S, Naik MN, Honavar SG: Orbital aspergillosis in immunocompetent patients. Br J Ophthalmol 2014, 98, 1379-1384.

[20] Breit SM, Hariprasad SM, Mieler WF: Management of endogenous fungal endophthalmitis with voriconazole and caspofungin. Am J Ophthalmol 2005, 139, 135-140.

[21] Silva RA, Sridhar J, Miller D, Wykoff CC, Flynn HW Jr: Exogenous fungal endophthalmitis: an analysis of isolates and susceptibilities to antifungal agents over a 20-year period (1990-2010). Am J Ophthalmol 2015, 159, 257-264.

[22] Riddell J, Comer GM, Kauffman CA: Treatment of Endogenous Fungal Endophthalmitis: Focus on New Antifungal Agents. Clin Infect Dis 2011, 52, 648-653.

[23] Mohamed MS, Abdel-Motaleb HY, Mobarak FA: Management of rhino-orbital mucormycosis. Saudi Med J 2015, $36,865-868$.

[24] Chahal HS, Abgaryan N, Lakshminarayanan R, Glover AT: Orbital Mucormycosis Following Periorbital Cutaneous Infection. Ophthal Plast Reconstr Surg 2015, Apr 7 [Epub ahead of print].

[25] Dzierżanowska D, Dzierżanowska-Fangrat K: Przewodnik terapii inwazyjnych zakażeń grzybiczych. $\boldsymbol{\alpha}$-medica Press, Bielsko-Biała 2010, 50-53.

\section{Address for correspondence:}

Monika Biernat

Department of Microbiology

Wroclaw Medical University

Chałubińskiego 4

Wrocław

Poland

Tel.: +48717841276

E-mail: mobiernat@gmail.com

Conflict of interest: None declared

Received: 25.09.2015

Revised: 27.10.2015

Accepted: 12.11.2015 